\title{
Parental Knowledge, Attitudes, and Behaviours towards Human Papillomavirus Vaccination for Their Children: A Systematic Review from 2001 to 2011
}

\author{
Kristina Trim, ${ }^{1}$ Naushin Nagji, ${ }^{1}$ Laurie Elit, ${ }^{2}$ and Katherine Roy $^{3}$ \\ ${ }^{1}$ Bachelor of Health Sciences Program, Faculty of Health Sciences, McMaster University, Hamilton, ON, Canada L8S 4L8 \\ ${ }^{2}$ Ontario Cervical Screening Program, Cancer Care Ontario and Division of Gynecologic Oncology, Juravinski Cancer Centre, \\ Hamilton, ON, Canada L8V 5C2 \\ ${ }^{3}$ Morden Street Research Services, Hamilton, ON, Canada L8S 4 S3
}

Correspondence should be addressed to Kristina Trim, trimks@gmail.com

Received 10 June 2011; Accepted 30 July 2011

Academic Editor: Jitti Hanprasertpong

Copyright (c) 2012 Kristina Trim et al. This is an open access article distributed under the Creative Commons Attribution License, which permits unrestricted use, distribution, and reproduction in any medium, provided the original work is properly cited.

\begin{abstract}
Objectives. A systematic review of parental surveys about HPV and/or child HPV vaccination to understand parental knowledge, attitudes, and behaviour before and after FDA approval of the quadrivalent HPV vaccine and the bivalent HPV vaccine. Search Strategy. Searches were conducted using electronic databases limited to published studies between 2001 and 2011 . Findings. The percentage of parents who heard about HPV rose over time (from 60\% in 2005 to $93 \%$ in 2009), as did their appreciation for the HPV infection and cervical cancer link (from 70\% in 2003 to $91 \%$ in 2011). During the FDA approval, there was a stronger vaccine awareness but it has waned. The same pattern is seen with parents whose children received the HPV vaccine (peak at $84 \%$ in 2010 and now 36\% in 2011) or the intention to vaccinate (peak at $80 \%$ in 2008 and now $41 \%$ in 2011). Conclusions. Parents had safety concerns and wanted more information their physician from to recommend and to confidently HPV vaccinate their children.
\end{abstract}

\section{Background}

Human papillomavirus (HPV) is the most common sexually transmitted infection in the world and is an established causative agent for cervical, anal, and penile cancers, as well as genital warts in both men and women $[1,2]$. It is estimated that $75 \%$ of Canadians will experience an HPV infection at least once in their lifetime, with the highest rates of infection occurring in individuals under the age of 25 [3]. In June 2006, the US Food and Drug Administration (FDA) approved the quadrivalent vaccine for use in the prevention of HPV strains 6,11,16, and 18, which are associated with $70 \%$ of cervical cancer and $90 \%$ of genital warts cases $[4,5]$. In October 2009, the bivalent HPV vaccine was approved by the FDA for the prevention of HPV strains 16 and 18 which are associated with $70 \%$ of cervical cancer cases $[4,6]$. Unlike the quadrivalent vaccine, the bivalent HPV vaccine does not protect against strains of HPV that cause genital warts [6]. Both vaccines are administered in three doses over a period of six months.
As a result of the approval of the HPV vaccines, recent health policy discussions have introduced the idea of adjusting the age of initial PAP smears from 18 years old (or with sexual debut) to 21 or 22 years old (or with sexual debut) [7]. Additionally, a move away from PAP smears toward HPV viral testing for women over 30 with a concurrent decrease in the frequency of PAP smear testing from annually to every 3 to 5 years has been proposed [7]. These health policy shifts are rooted in the success of the HPV vaccines to guard against cervical cancer. This success is, necessarily, dependent on successful vaccine uptake.

Currently, policy is modelled on an $80 \%$ uptake by young women, which means when combined with vaccination, reducing the frequency of testing and increasing the age of initial PAP smear would be part of an efficient plan to reduce cervical cancer. However, actual uptake of the vaccines is relatively low and not consistent in all areas that the vaccine is offered. For example, in the province of Quebec where there is a passive consent strategy to school immunizations (i.e., parental consent must be explicitly withdrawn in a note 
to the school), there is an $80 \%$ vaccine uptake for grade- 8 girls $[8,9]$. However, in the province of Ontario, where the school-based immunization program has an active consent strategy (i.e., parental consent is explicitly given in a note to the school), the vaccine uptake rate for grade 8-girls is $50 \%[8,9]$. By comparison, the acceptance of the hepatitis $B$ vaccine was accepted without difficulty yet both aim at preventing disease that is sexually acquired. The acceptance of the hepatitis B vaccine for grade-7 students in Ontario was $79.8 \%$ (range $65.2 \%$ to $95.2 \%$ ) and in Quebec the acceptance for grade- 8 students was between 85 and 95\% $[8,9]$. The hepatitis vaccine is offered to both boys and girls and is marketed to prevent liver disease and liver cancer, which is relatively rare in the developed world compared to cervical cancer. Suggested reasons for a low vaccine uptake rate range from low knowledge levels regarding HPV and the HPV vaccine, to cost, to a perceived low efficacy of the vaccine. Recent literature has examined these possible factors as they relate to adolescent attitudes towards HPV vaccination [7]. However, given that the vaccines are targeted towards males and females in the 9- to 26-age group, with emphasis placed on ages 11 and 12 in order to promote inoculation prior to sexual debut [10], a key factor in the implementation of HPV vaccines is the extent to which parents accept HPV vaccination for their children. In order to fully understand the issues surrounding HPV vaccine uptake, parental attitudes towards the vaccine must be examined.

\section{Objectives}

The purpose of this systematic paper is to compare the findings of previous studies that have examined parental knowledge, attitudes, and behaviours towards the HPV vaccine. Particular emphasis will be placed on changes within parental knowledge, attitudes, and behaviour following the availability of the HPV vaccines. Beyond identifying trends in uptake, the paper will also focus on factors that affect parental intentions to vaccinate their children against HPV. Based on previous studies regarding vaccination, these factors may include parental knowledge regarding cervical cancer and STIs (i.e., genital warts); perceived risk and severity of cervical cancer and genital warts; attitudes towards vaccines in general; issues concerning increase in sexual activity or promiscuity; availability of health insurance to cover vaccine costs. Additionally, a preliminary analysis of parental attitudes towards STI-prevention interventions versus anticancer interventions will be conducted to determine the policy implications of the two vaccines; one of which is part of an anticancer strategy as well as STI (genital warts) prevention and one of which only targets cervical cancer prevention.

\section{Method}

3.1. Search Strategy. Prior to conducting the literature search, a librarian was consulted for assistance in building a comprehensive search strategy. Relevant research studies were located through an extensive search of the electronic databases PubMed, Ovid MEDLINE, Embase, and
Cumulative Index of Nursing and Allied Health Literature (CINAHL). Searches were limited to only those studies which were published between 2001 and 2011 in an effort to obtain the most recent articles regarding parental knowledge, attitudes, and behaviour before and after FDA approval of the quadrivalent $H P V$ vaccine and the bivalent HPV vaccine.

A preliminary hand search of the literature was completed in order to identify appropriate keywords and medical subject heading (MESH) terms. The terms that were selected to be used in this paper were "HPV", "parent" and "vaccine," with "parent-child relations," "papillomavirus vaccine," "parent or child parent relation," and "wart virus vaccine" often being utilized as synonyms. Search terms were combined using the operators "AND" and "OR" to ensure that all relevant articles were located.

3.2. Study Selection. A total of 325 articles were identified: 304 from the initial search strategy and an additional 21 articles were gathered from the hand search of the literature. Following the removal of duplicates $(n=71), 254$ articles were screened for inclusion in the paper (Figure 1). Inclusion criteria included (a) the study was about parents and their attitudes towards HPV and/or HPV vaccination; (b) the report had cross-sectional data about the parent's knowledge, attitudes, or beliefs about HPV that were not previously influenced by the research team with an intervention. Exclusion criteria included (a) sample population was not comprised of parents; (b) knowledge, attitudes, and/or behaviours of parents not discussed in results; (c) methodology of study did not include survey; (d) article was not based on original research (i.e., the study was a literature review); (e) the full article was not available in English. The results of the study selection process are shown in Figure 1.

3.3. Data Collection and Abstraction. The data abstraction form was created and was pilot tested on five articles. Once the remaining modifications were made to the abstraction form, three coders extracted the data based on the coding information provided on the form. The data was then entered into SPSS for analysis, with information being transformed into percentages where possible. Initially, five outcomes that correspond to parental knowledge, attitudes, or behaviours were recorded. These five outcomes were that the parents had heard of HPV, heard of the HPV vaccine, knowledge of association between HPV and cervical cancer, an intention to vaccinate their child, and vaccinated their child(ren) with one or more doses of the vaccine. Following this initial analysis, an analysis of factors affecting parental attitudes toward the vaccines was conducted.

\section{Results}

4.1. Characteristics of Study Samples. The literature search resulted in 53 studies that met inclusion criteria and were included in this systematic paper [11-63]. All included studies have been listed by publication date, research question, and focus in Figure 4. Publication dates were between the years of 2004 and 2011 with the majority of the studies being published in 2009 (28.3\%) and 2010 (34.0\%). Surveys 


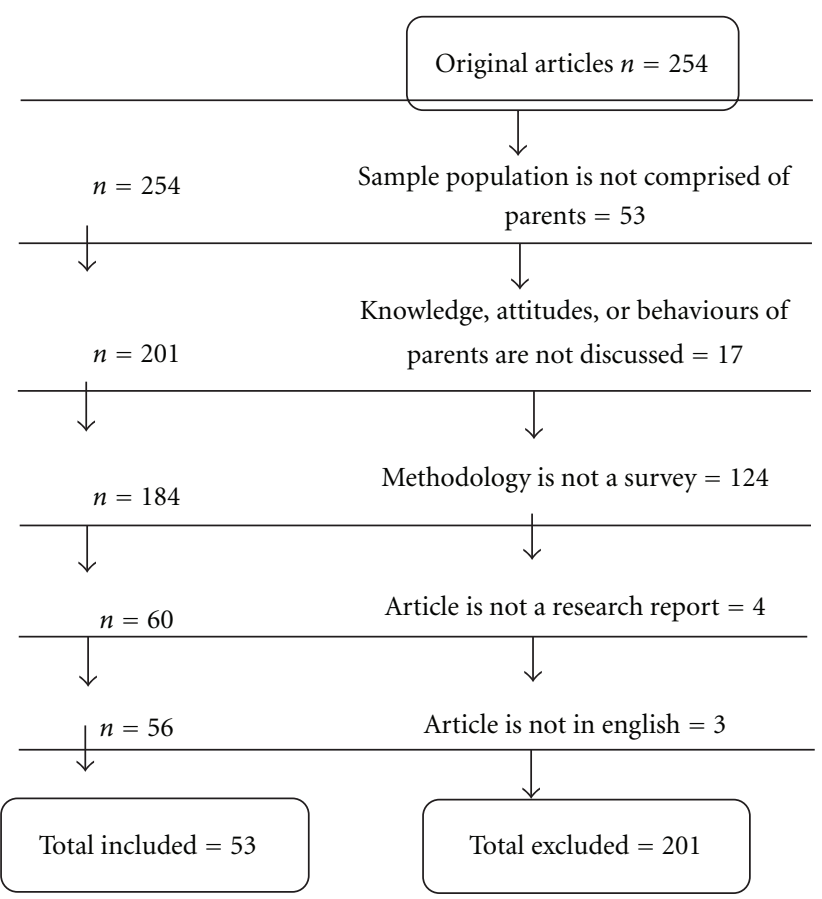

FIGURE 1: Inclusion and exclusion process.

were administered to parents in 2007 or earlier in $60.4 \%$ of the studies. The majority of the studies were conducted in North America (USA: 56.6\%; Canada: 3.8\%), however, the European Union (24.5\%), Asia (9.4\%), and New Zealand or Australia (5.7\%) were also represented. Figure 2 highlights the geographic representation of the sample. Forty-one percent of studies were conducted in a school or medical setting, while $45.3 \%$ used some form of population-based sampling (e.g., census or government data, random digit dialling, or an existing longitudinal study), and 13.2\% used other sampling procedures. These "other" sampling procedures included pretest data from an educational HPV intervention or mixed methods. Figure 3 demonstrates the methodology of the studies in the sample.

The total number of parents included in this study was 54,194 with a median study sample size of 506, and a mean study sample size of $1,022(\mathrm{SD}=2,099)$. Six studies only reported "parents" and did not differentiate between mothers and fathers. Twenty-three studies (43\%) reported mothers' responses only. Of those studies that reported both mothers' and fathers' attitudes, the majority of respondents were mothers with the average sample size of mothers at 82.3\% (minimum: $47.7 \%$ and maximum: $95.1 \%$ ).

4.2. Framework for Analyses. Studies posed a wide variety of research questions; in order to simplify analyses, each study's central question and results were grouped according to whether they were concerned with parental knowledge of HPV, parental behaviours toward the HPV vaccines, parental intent to vaccinate their children against HPV (attitudes), or a combination of the three factors (Table 1 ).

Of the 53 studies included, $73.6 \%$ (39/53) attempted to gauge parental knowledge of HPV and the HPV vaccine.

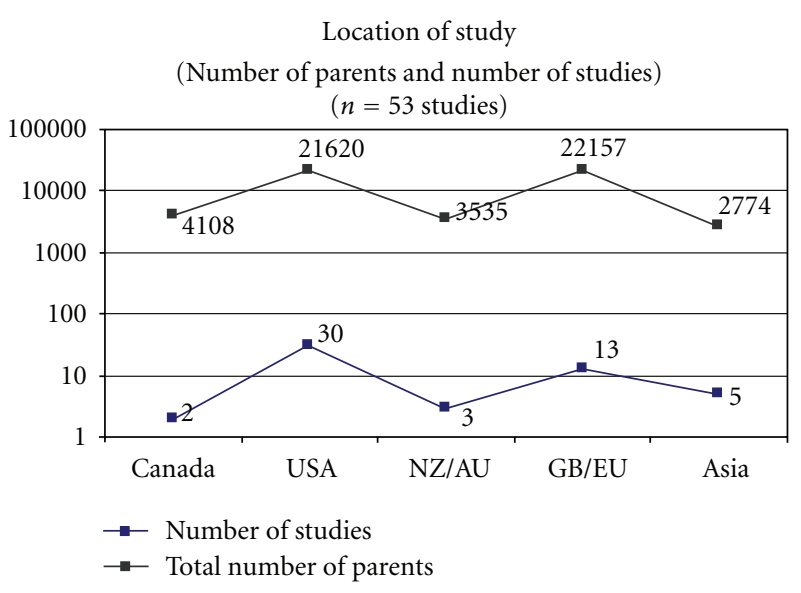

FIgURe 2: Location of study.

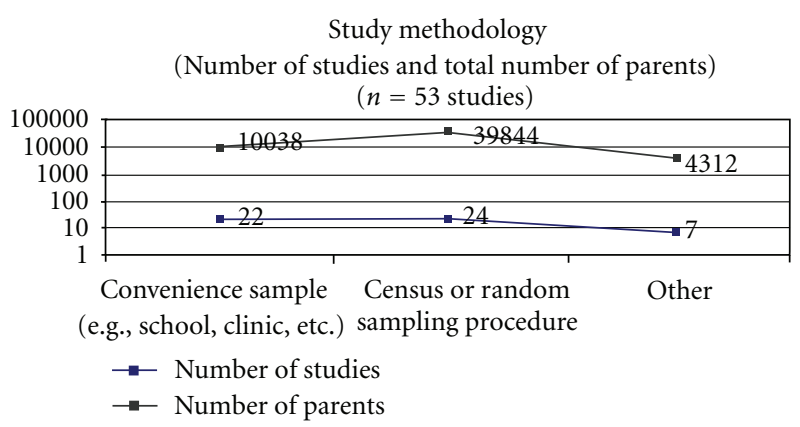

FIgUre 3: Study methodology.

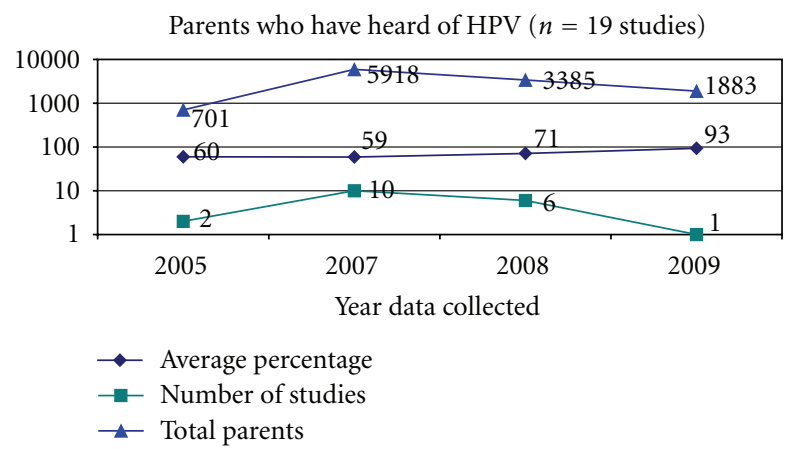

Figure 4: Heard of HPV.

This ranged from whether parents were aware of HPV to whether parents could correctly identify HPV as the causative agent of cervical cancer. Thirty-eight percent (20/53) of studies focused on parental behaviour (i.e., whether parents had already inoculated their child or children against HPV with either of the two vaccines available). Finally, 92.5\% (49/53) of studies focused on parental attitudes toward the vaccine (whether parents intend to vaccinate their children against HPV). Many studies also focused on factors affecting parental attitudes and behaviour regarding the HPV vaccine. These factors included perceived vaccine efficacy; vaccine safety; perceived threat of HPV. They will be discussed more thoroughly in the section regarding factors and barriers. 


\begin{tabular}{|c|c|c|c|c|c|c|c|c|c|c|}
\hline $\mathscr{\mathscr { X }}$ & $\mathscr{ֶ}$ & $\mathscr{ֶ}$ & 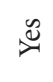 & $\Leftrightarrow$ & $\mathscr{\mathscr { N }}$ & $\check{્}$ & $\cong$ & $\stackrel{\circ}{2}$ & $\mathscr{\approx}$ & $\approx$ \\
\hline 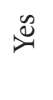 & ż & ż & z & $\bar{z}$ & z̊ & $\mathscr{ٍ ్ ర ~}$ & $\stackrel{2}{2}$ & 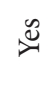 & $\stackrel{2}{z}$ & $\check{\circlearrowright}$ \\
\hline
\end{tabular}

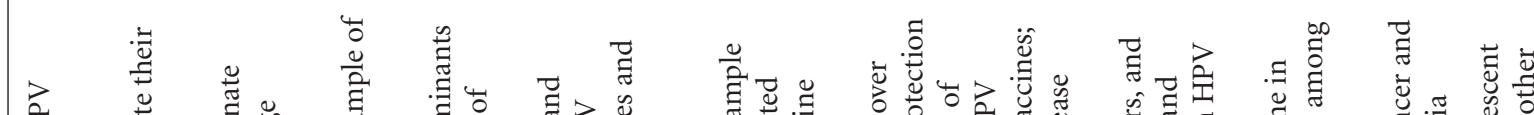

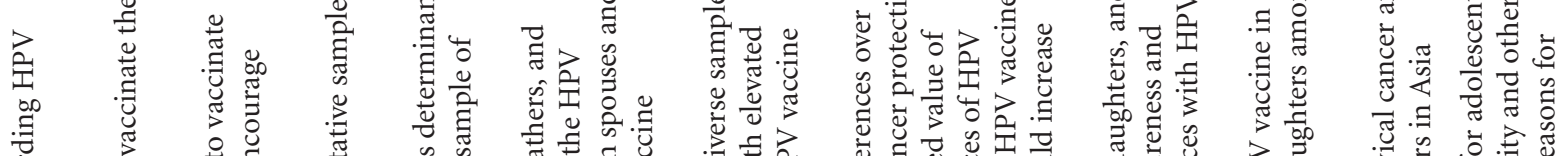

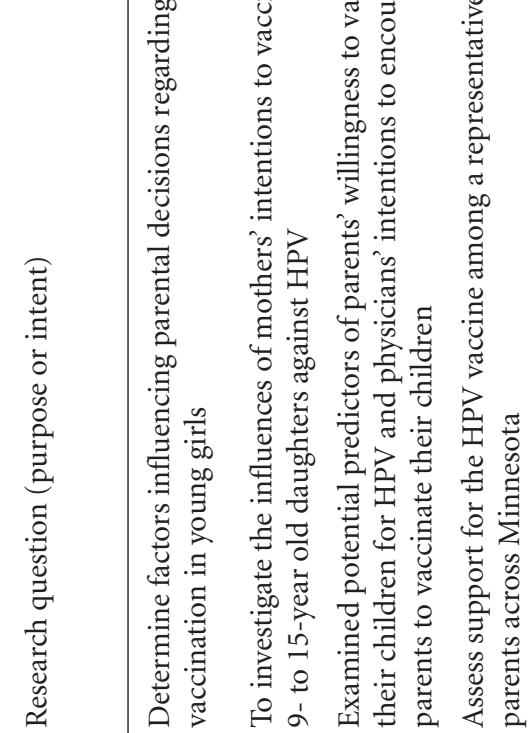

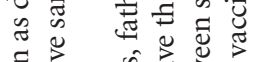

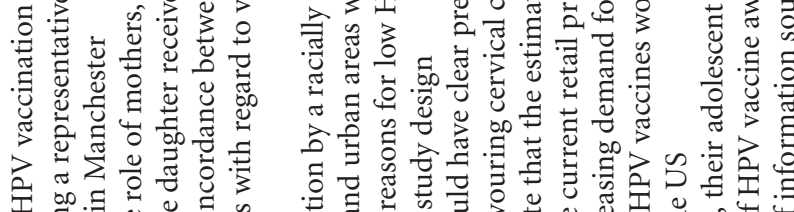

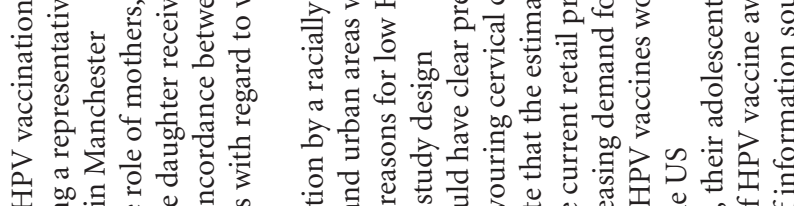

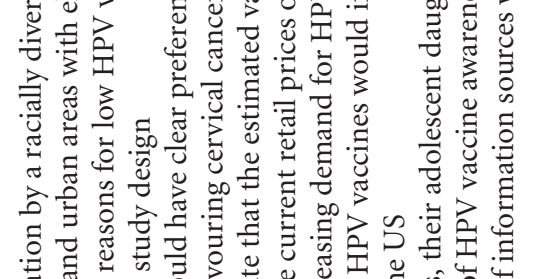

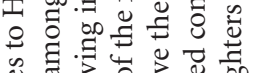

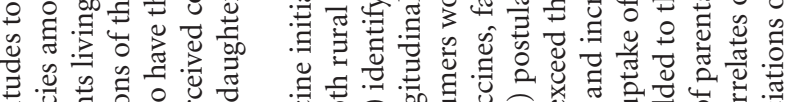

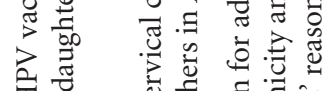

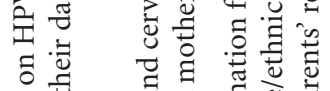

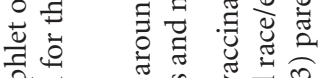

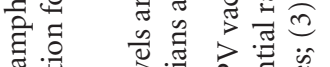

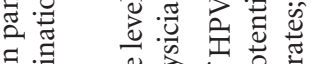
. 矛

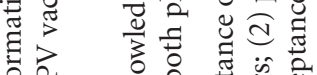

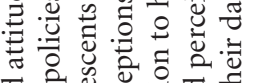

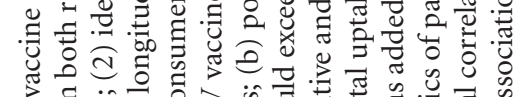

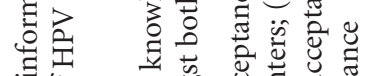

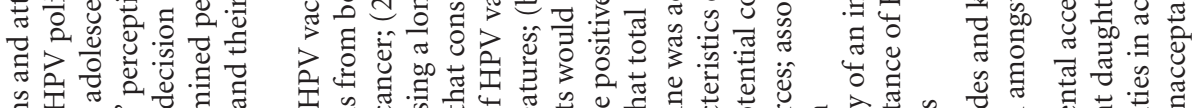

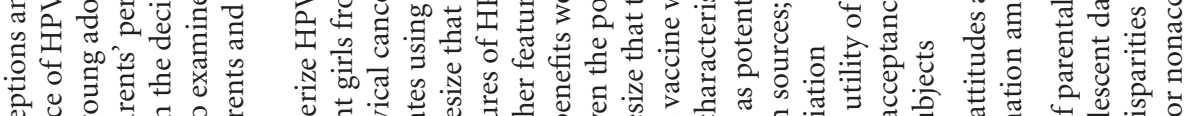

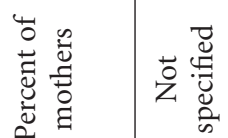

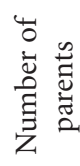

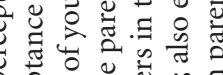

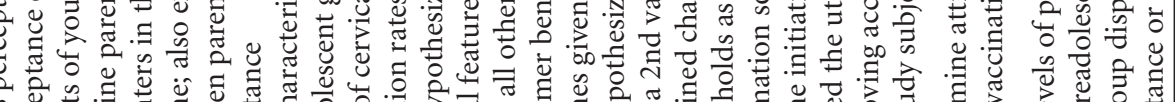

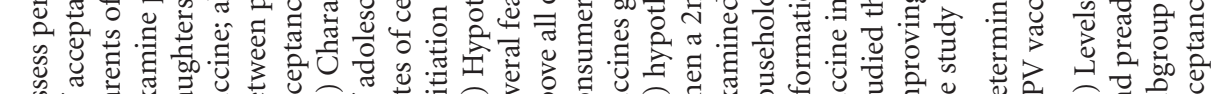

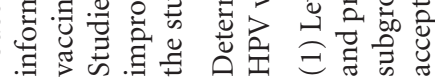

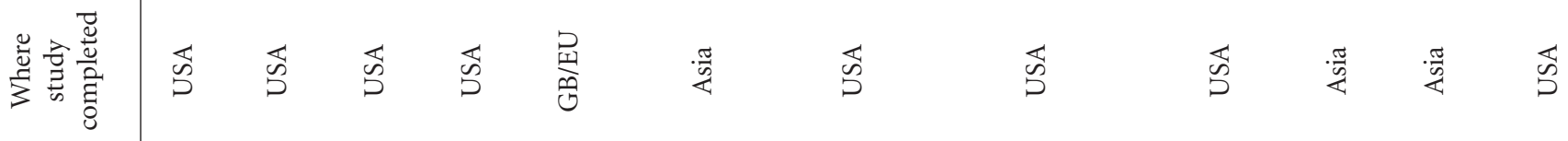

के 节: ڤิ) .ำำ 焉

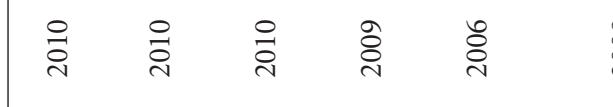
ᄋे $\stackrel{2}{2}$ 웅 유 章

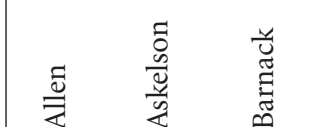

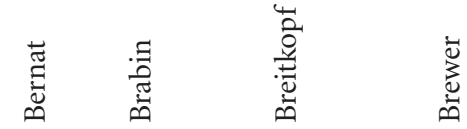
芯苛苛 葛

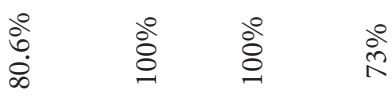
จำ ถิ

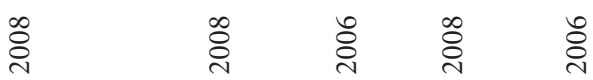
营 


\begin{tabular}{|c|c|c|c|c|c|c|c|c|c|c|c|c|c|c|}
\hline 胥起 & $\mathscr{x}$ & $\cong$ & 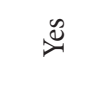 & $\mathscr{\swarrow}$ & $\cong$ & $\dddot{̋}$ & $\cong$ & $\approx$ & 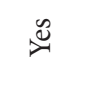 & $\mathscr{্}$ & $\cong$ & $\mathscr{\mho}$ & $\mathscr{્}$ & 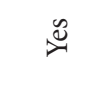 \\
\hline 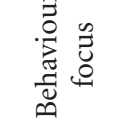 & $\stackrel{8}{2}$ & $\stackrel{\circ}{z}$ & $\stackrel{2}{z}$ & $\AA$ & $\mathscr{\mho}$ & ஜ & $\stackrel{2}{2}$ & ஜ & $\approx$ & $\cong$ & 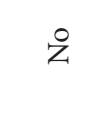 & $\mathscr{*}$ & $\cong$ & \&̊ \\
\hline 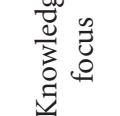 & 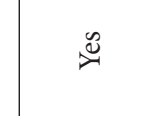 & $\cong$ & $\approx$ & $\cong$ & $\cong$ & 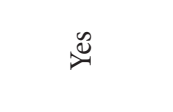 & $\cong$ & $\cong$ & $\approx$ & 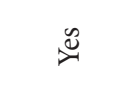 & $\cong$ & $\cong$ & $\cong$ & $\cong$ \\
\hline 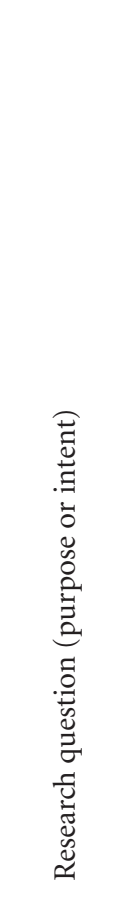 & 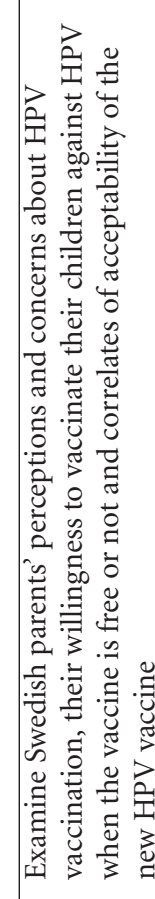 & 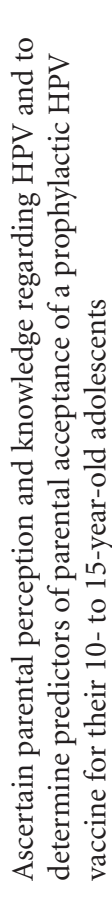 & 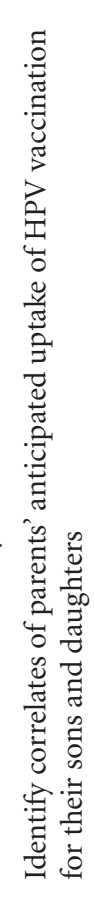 & 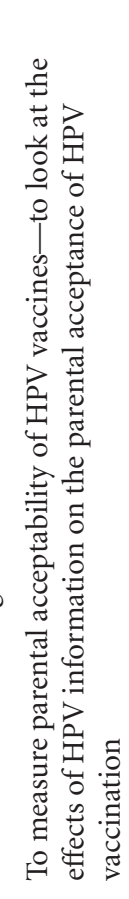 & 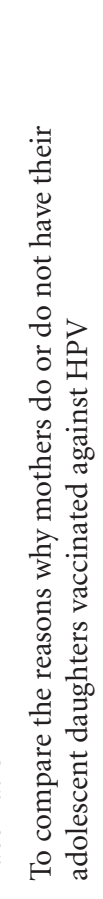 & 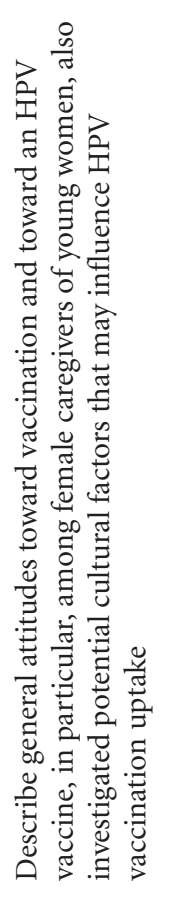 & 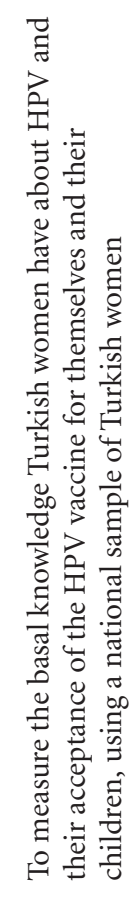 & 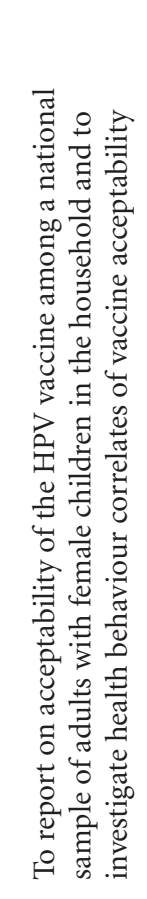 & 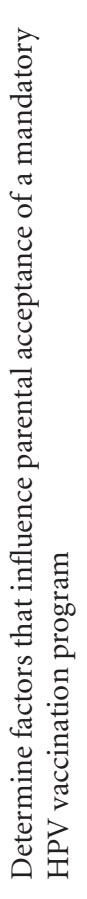 & 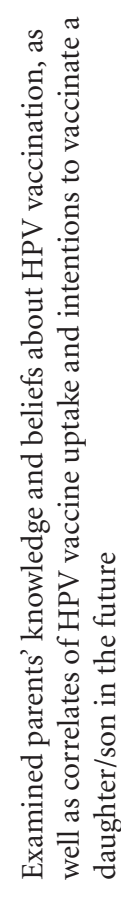 & 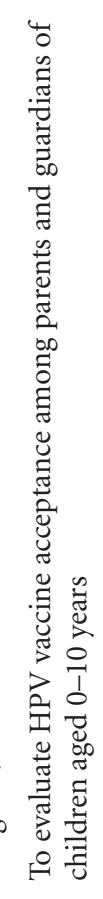 & 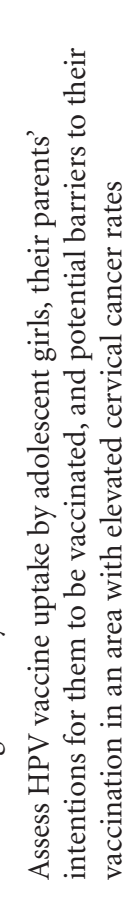 & 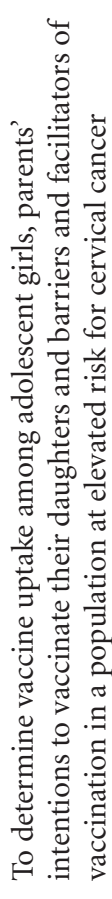 & 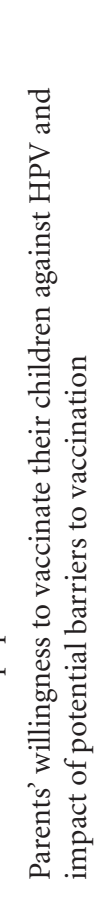 \\
\hline 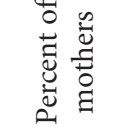 & $\stackrel{\circ}{\circ}$ & $\stackrel{\circ}{\circ}$ & 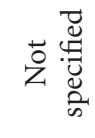 & 广্े & 㤐 & 总 & 宮 & $\begin{array}{c}\stackrel{\circ}{0} \\
\stackrel{\infty}{i} \\
i n\end{array}$ & 京 & ڤ̀ & 遖 & ळे & 遖 & 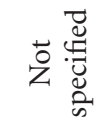 \\
\hline 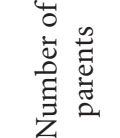 & $\begin{array}{l}0 \\
\text { ț } \\
\text { d }\end{array}$ & 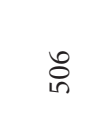 & ஜ్ల & $\exists$ & in & $\stackrel{\infty}{=}$ & I & $\stackrel{\infty}{\stackrel{\infty}{2}}$ & $\stackrel{\text { }}{\sim}$ & $\infty$ & $\vec{\infty}$ & $\begin{array}{l}\stackrel{a}{\infty} \\
\infty\end{array}$ & :े & 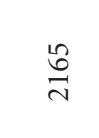 \\
\hline 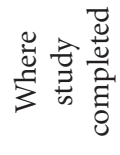 & $\begin{array}{l}\text { P्य } \\
\text { 㺃 }\end{array}$ & 峁 & 苗 & 芯 & $\vec{s}$ & 采 & 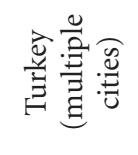 & 范 & $\overleftrightarrow{b}$ & 芯 & $\overleftrightarrow{\Delta}$ & 苑 & $\overleftrightarrow{b}$ & $\frac{\text { Z्t }}{\mathrm{N}}$ \\
\hline 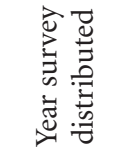 & ڤ్ & ڤ్రి & 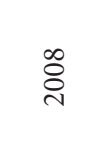 & 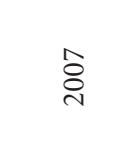 & 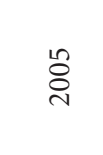 & 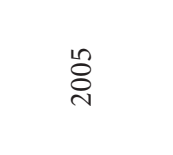 & ثे & 今ે & 完 & $\stackrel{\text { ¿ }}{\infty}$ & $\overrightarrow{\vec{N}}$ & ڤ్ & ڤે & 苍 \\
\hline 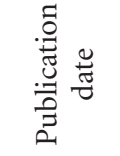 & ذ્సे & 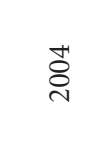 & $\stackrel{\infty}{\stackrel{\circ}{0}}$ & 崩 & ఫ्रे & ڤ্ণ & ఫे & 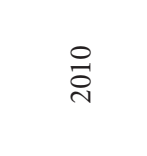 & ते & ఫे & $\overrightarrow{\vec{i}}$ & ఫे & $\overrightarrow{\mathrm{N}}$ & ثे \\
\hline 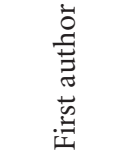 & 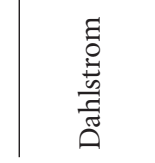 & 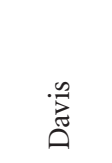 & 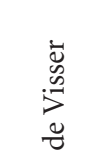 & 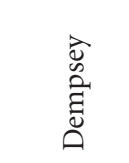 & 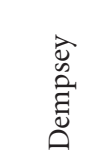 & 氞 & 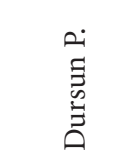 & 点 & $\overrightarrow{\vec{W}}$ & $\begin{array}{l}\vec{\Xi} \\
\text { 节 }\end{array}$ & 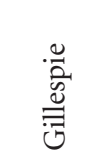 & 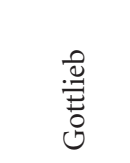 & 壳 & 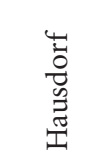 \\
\hline
\end{tabular}




\begin{tabular}{|c|c|c|c|c|c|c|c|c|c|c|c|c|c|}
\hline 吾总 & $\cong$ & $\stackrel{2}{2}$ & 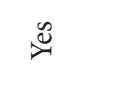 & $\cong$ & $\mathscr{\mho}$ & $\dddot{\mathscr{x}}$ & 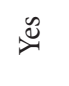 & $\cong$ & $\mathscr{\varkappa}$ & $\mathscr{\simeq}$ & 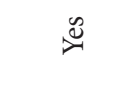 & 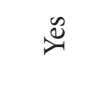 & $\mathscr{্}$ \\
\hline 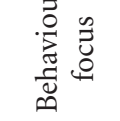 & 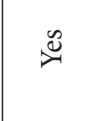 & 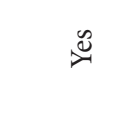 & z̊ & $\stackrel{\circ}{2}$ & z̊ & $\stackrel{2}{z}$ & $\stackrel{\circ}{2}$ & $\stackrel{8}{z}$ & $\stackrel{\circ}{z}$ & $\stackrel{\circ}{z}$ & $\cong$ & 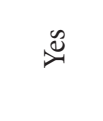 & $\stackrel{\circ}{z}$ \\
\hline 丞 & $\check{્ ゙ ~}$ & 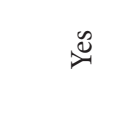 & 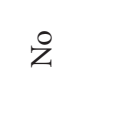 & z̊ & $\triangleq$ & $\dddot{\varkappa}$ & $\stackrel{\circ}{2}$ & $\stackrel{\circ}{z}$ & 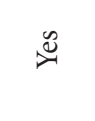 & z & ż & $\cong$ & 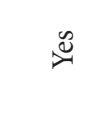 \\
\hline 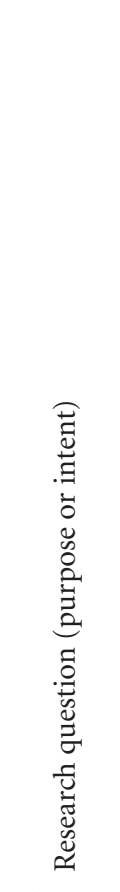 & 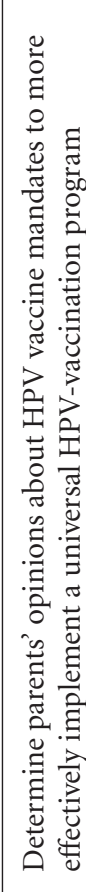 & 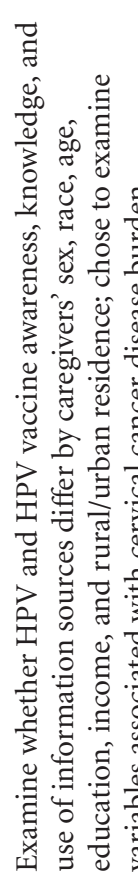 & 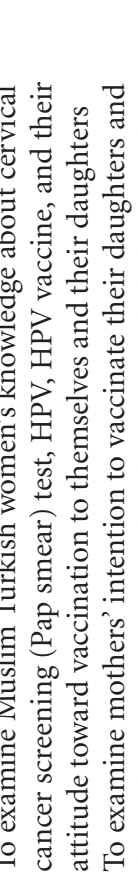 & 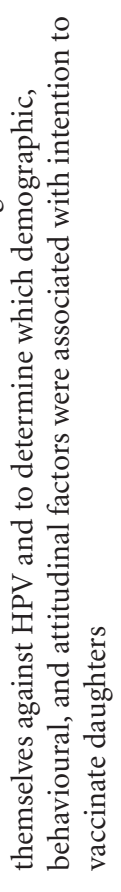 & 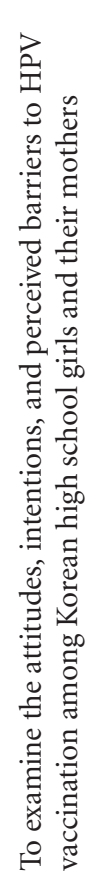 & 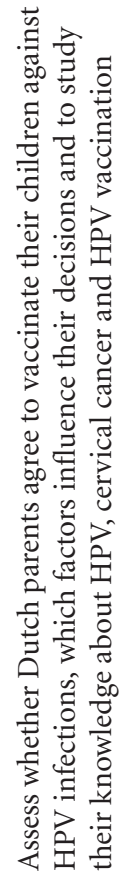 & 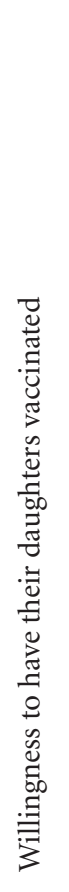 & 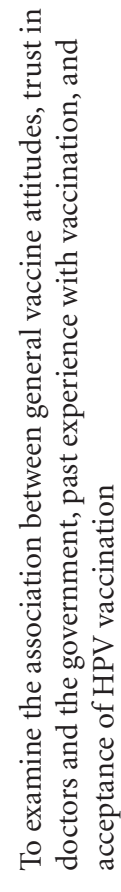 & 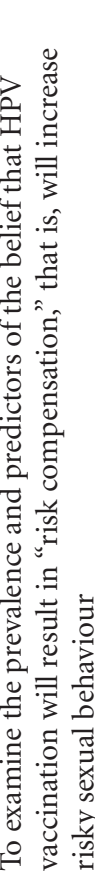 & 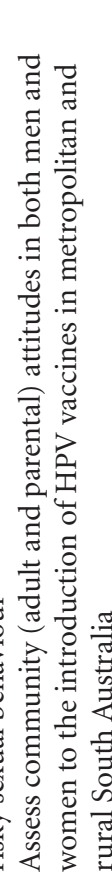 & 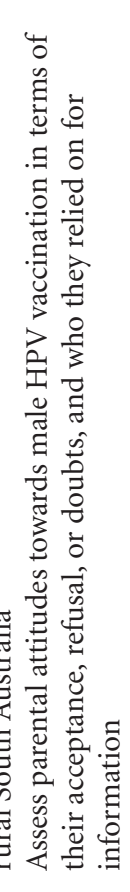 & 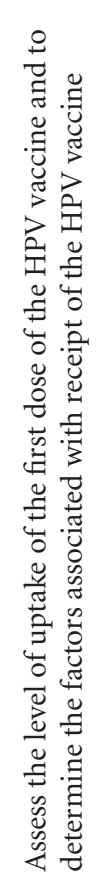 & 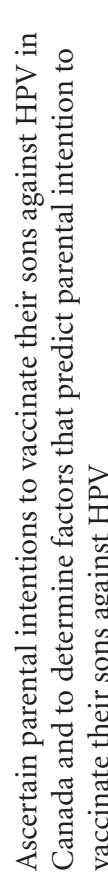 \\
\hline 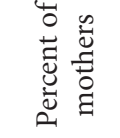 & $\begin{array}{l}0 \\
\dot{0} \\
\infty \\
\infty \\
\infty\end{array}$ & ओें & 高 & 。̊̊̊口 & 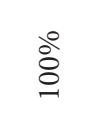 & $\stackrel{\circ}{\circ}$ & $\stackrel{\circ}{\circ}$ & 宮 & ڤ̊ํㅇ & 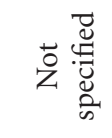 & ฌे & $\begin{array}{l}\stackrel{0}{\circ} \\
\dot{+} \\
\dot{\infty}\end{array}$ & 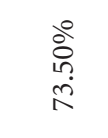 \\
\hline 壳 & $\stackrel{\sim}{\sim}$ & $\mathscr{\infty}_{\infty}$ & $\vec{m}$ & ڤે & : & 吕 & ஃั & 范 & $\tilde{m}$ & $\bar{b}$ & 字 & ڤ్రి & $\stackrel{\infty}{\stackrel{\sim}{0}}$ \\
\hline 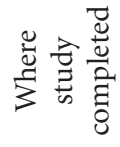 & 芯 & $\overleftrightarrow{\Delta}$ & $\begin{array}{l}\text { P } \\
\text { 焉 }\end{array}$ & 崩 & $\frac{9}{8}$ & $\begin{array}{l}\text { 总 } \\
\text { 兽 }\end{array}$ & 总 & 总 & 邑 & $\frac{?}{\mathrm{~N}}$ & 总 & 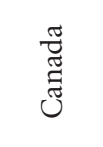 & $\begin{array}{l}\text { 䔍 } \\
\text { 芯 }\end{array}$ \\
\hline 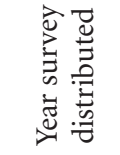 & 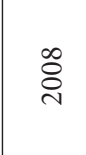 & 商 & ڤ્તે & ڤ్రે & ذ્ণे & 商 & 总 & 商 & : & : & :ें & ఫ्रे & ثे \\
\hline 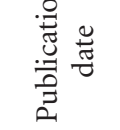 & ठ্ণે & ఫ్సి & 옴 & ఫ్రి & ڤ̊ํํ & 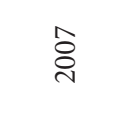 & 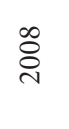 & ث્ণ & ذ્సે & ثે & :े & :े & ڤ్ \\
\hline 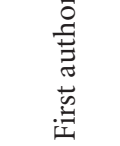 & 志 & 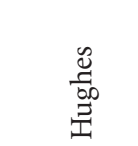 & $\stackrel{\ddot{\Xi}}{=}$ & 胥 & $\begin{array}{l}\text { 点 } \\
\text { 吨 }\end{array}$ & 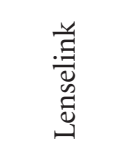 & $\begin{array}{l}\frac{3}{0} \\
\frac{0}{5} \\
\frac{1}{2}\end{array}$ & $\begin{array}{l}\frac{3}{0} \\
\frac{0}{\pi} \\
\frac{\pi}{2}\end{array}$ & $\begin{array}{l}\frac{3}{0} \\
\frac{0}{\pi} \\
\frac{\pi}{2}\end{array}$ & 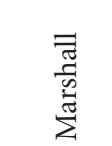 & 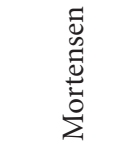 & 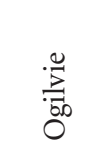 & 莺 \\
\hline
\end{tabular}




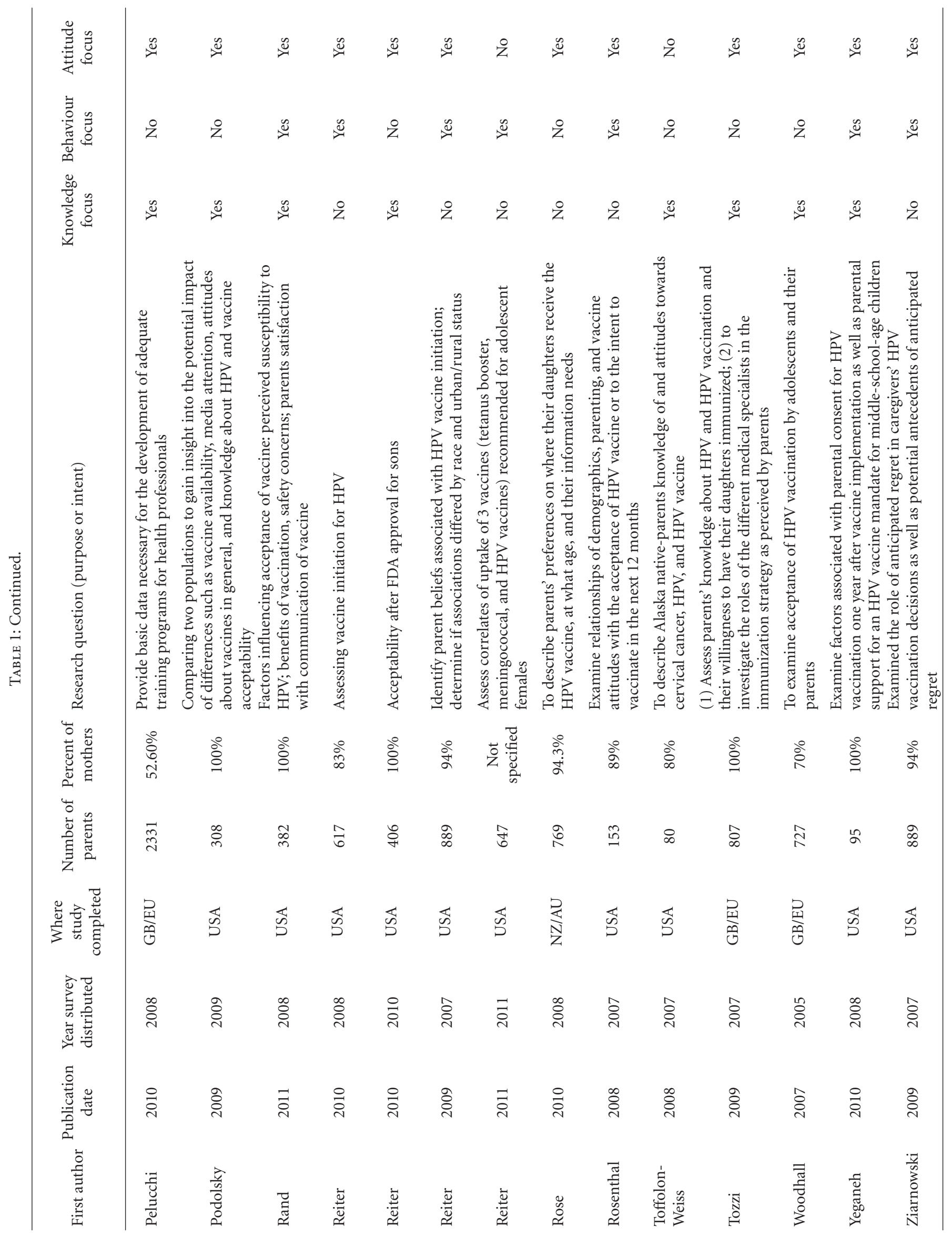


4.3. Knowledge Trends. Three primary knowledge questions were examined: whether parents had heard of HPV, whether they had heard of the HPV vaccine, and whether they could correctly identify the relationship between HPV and cervical cancer. Of the 53 studies, 19 studies (36\%) asked parents whether they had heard of HPV prior to being included in the study (Figure 4).

Parental awareness of HPV increased in 2008 and 2009. Of the 53 studies, 15 studies $(28 \%)$ asked parents whether they had heard of the HPV vaccines prior to being included in the study (Figure 5).

Parental awareness of the HPV vaccine spiked in 2007 with a mean percentage of 59\% compared to 14\% in 2006 . Awareness continued to climb to $65 \%$ in 2008 and dropped off slightly to $47 \%$ by 2010 . These years are of particular interest since they mark the introduction and availability of the quadrivalent $\mathrm{HPV}$ vaccine and the bivalent $\mathrm{HPV}$ vaccine. Of the 53 studies, 5 studies (9\%) asked parents if they could identify the relationship between cervical cancer and HPV (Figure 6).

It is important to note that only 5 studies asked parents to make the connection between HPV and cervical cancer. In the study in which data were collected most recently (2011), an average of $74 \%$ of parents could correctly identify the relationship between HPV and cervical cancer. With only 5 studies examining parental knowledge of the relationship between HPV and cervical cancer, it is difficult to make any connections between knowledge and the introduction and availability of the HPV vaccine.

4.4. Behaviour Trends. Of the 53 studies, 17 studies (32\%) asked parents whether their child or children had already been vaccinated against HPV (Figure 7).

Following the availability of the quadrivalent HPV vaccine in 2007, studies began asking whether parents had already vaccinated their children against HPV. The highest percentages of parents who had vaccinated their children against HPV occurred in 2009 and 2010. This is following the introduction and availability of the bivalent $\mathrm{HPV}$ vaccine in 2009 .

4.5. Attitude Trends. Of the 53 studies included, 30 studies (57\%) asked parents whether they intend to vaccinate their child or children against HPV (Figure 8).

The highest percentage of parents who intend to vaccinate their children $(86 \%)$ occurs in studies where the data were collected in 2005, prior to the release of the first HPV vaccine. Intent increases in 2008 to $80 \%$ of parents from $67 \%$ in 2007 and then gradually decreases in 2009, 2010, and 2011.

All three knowledge components have increased from pre-2007 studies to post-2007 studies. While levels of uptake pre-2007 and post-2007 cannot be compared, intent to vaccinate has decreased from pre-2007 to post-2007 (Table 2).

4.6. Factors Affecting Parental Decision to Vaccinate. Of the 53 studies included, $81 \%$ made some mentioning of examining barriers to parental intent to vaccinate. Parental experiences and demographic characteristics were too mixed to show any

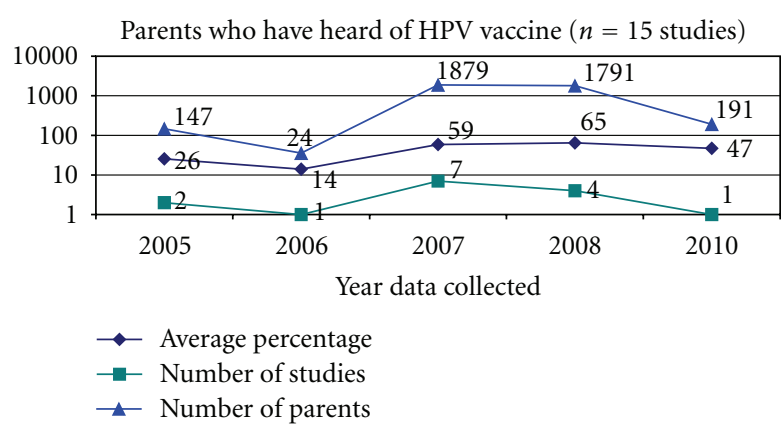

FIGURE 5: Heard of HPV vaccines.

Parents who understood connection between HPV and cervical cancer

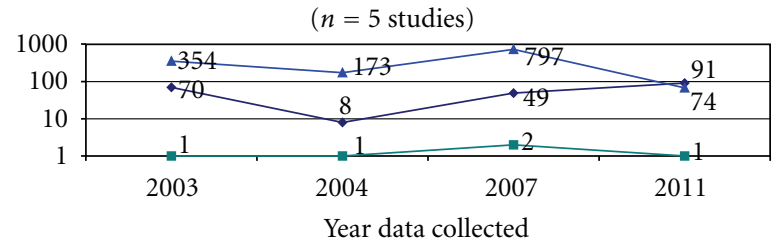

$\rightarrow$ Average percentage

$\rightarrow$ Number of studies

$\neg$ Number of parents

FIgURE 6: Cervical cancer and HPV.

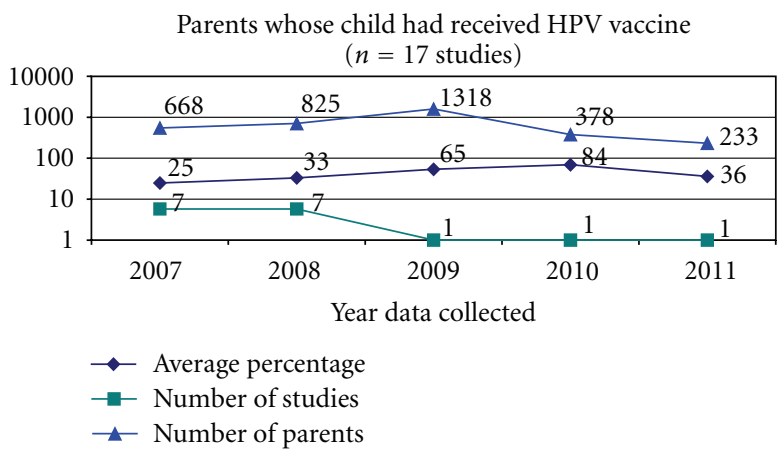

FIgURE 7: Received HPV vaccine.

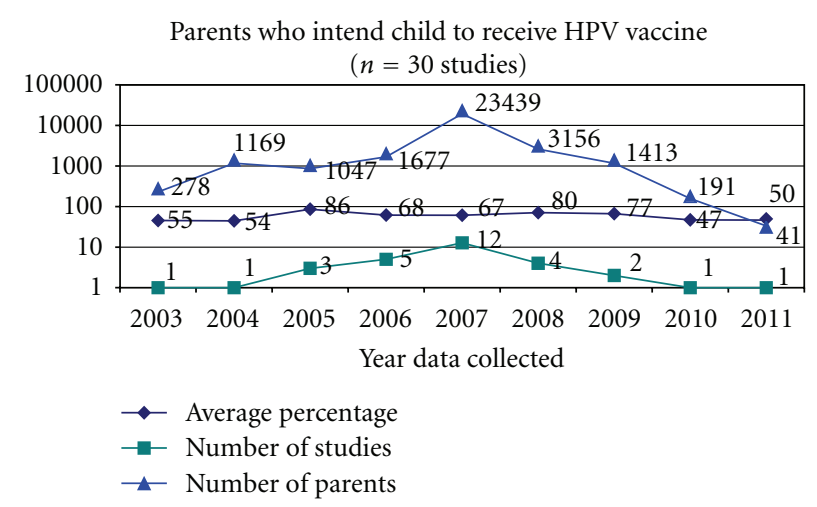

FIGURE 8: Intent to inoculate against HPV. 
TABLE 2: Summary of trends prior to 2007 and after 2007.

\begin{tabular}{|c|c|c|c|c|c|c|}
\hline & \multirow{2}{*}{$\begin{array}{c}\text { Number of studies } \\
2007 \text { or earlier }\end{array}$} & \multicolumn{2}{|c|}{ Range values 2007 or earlier } & \multirow{2}{*}{$\begin{array}{c}\text { Number of studies } \\
2008 \text { or later }\end{array}$} & \multicolumn{2}{|c|}{ Range values 2008 or late } \\
\hline & & $\min \%$ & $\max \%$ & & $\min \%$ & $\max \%$ \\
\hline Heard of HPV & 12 & 59.0 & 59.5 & 7 & 64.7 & 93.0 \\
\hline Heard of HPV vaccine & 10 & 14.0 & 58.7 & 5 & 47.0 & 64.5 \\
\hline $\begin{array}{l}\text { Understood connection } \\
\text { between HPV and } \\
\text { Cervical Cancer }\end{array}$ & 4 & 8.0 & 70.0 & 1 & 53.4 & 91.0 \\
\hline $\begin{array}{l}\text { Parent intends to } \\
\text { vaccinate child }\end{array}$ & 22 & 54.0 & 86.0 & 8 & 47.0 & 79.5 \\
\hline Child is vaccinated HPV & NA & NA & & 17 & 24.9 & 84.0 \\
\hline
\end{tabular}

clear pattern within the 53 studies. Cost factors were also mentioned, but were difficult to compare across studies.

\subsection{Knowledge}

Parents Concerned about the Safety of the HPV Vaccine. In 20 studies (37\%), parents expressed concerns about vaccine safety and the potential side effects of the HPV vaccine $[13,15,20,23,30,32,33,35,37,39,41,42,46-48,52-$ $54,60,61]$.

Parents Wanted More Information about the Vaccine to Make an Informed Decision as to Whether They Should Vaccinate Their Child with the HPV Vaccine. In 13 studies (25\%), parents needed more information about HPV vaccination $[17,30,34,41,42,46-48,51,52,54,60,62]$.

\subsection{Attitudes}

Parents Who Were Concerned about the Potential Risk of Cancer Were More Likely to Vaccinate for HPV. In 16 studies $(30 \%)$, parents mentioned a concern about cancer risk as increasing the likelihood of HPV vaccination. Parents who believed it was likely that their daughters might contract HPV $[13,15,18,31-33,50,52]$, develop cervical or penile cancer $[13,18,20,30,31,39,47,50,53,60,62,63]$ or genital warts $[13,18,47,62]$ were more likely to vaccinate their daughters.

Parents Agreed That Children Should Be Older and Sexually Active to Receive the Vaccine. There were 10 studies (19\%) that mentioned the child's age affecting the parents' decision to vaccinate. Parents were less likely to vaccinate their children if they believed their children were too young or not sexually active [34, 40,44, 48]. Some studies indicated that parents were more likely to vaccinate their children if they were sexually active [23] or older [32, 34, 53-55, 57].

Mixed Opinions About Parental Concerns for Increased or More Risky Sexual Activity If Child Is Vaccinated. Parents were not concerned that their children would become sexually active if they were given the HPV vaccine in 13 studies $(25 \%)$ [ $12,14,17,28,32-34,39,42,46,51,60,62]$, while in six studies $(13 \%)$, parents expressed concerns that the HPV vaccine might encourage earlier sexual initiation, or more risky sexual behaviours in their children $[15,23,43$, $48-50,61]$.

\subsection{Behaviours}

Parents Looked to Their Physicians to Recommend the HPV Vaccine. In 17 studies (32\%), parents indicated that having their doctors recommend the vaccine increased the likelihood of HPV vaccination $[17,19,24,28,30-35,41,47,51-$ $53,60,62]$.

Parental Attitudes towards Vaccines Generally Indicated Whether They Were in Favour of Vaccinating Their Children for $H P V$. Parents who had previously vaccinated their children against meningitis [19] or had a general belief in the efficacy of vaccines $[15,24,25,32,45,48-50,62]$ were more likely to vaccinate (10 studies or $19 \%$ ). Parents who had refused previous vaccines for their children $[11,18,45,47$, $63]$ and had concerns about too many vaccinations $[45,60]$ were less likely to vaccinate (6 studies or $11 \%$ ).

\section{Discussion}

This paper reviews the parental knowledge, attitudes, and behaviours toward having their daughters and sons vaccinated against cervical cancer. The parents in these studies were largely from a high resource background. The percentage of parents that participated in these surveys who had heard about HPV clearly rose over time (from 60\% in 2005 to $93 \%$ in 2009). Parents' appreciation for the link between HPV infection and cervical cancer did rise $(70 \%$ in 2003 to $91 \%$ in 2011). During the era of FDA approval of the vaccines, there appeared to be stronger awareness of the vaccines and this has waned with time. This same pattern is seen with the percentage of parents whose children had received the HPV vaccine (high of $84 \%$ in 2010 and now $36 \%$ in 2011). Unfortunately, this pattern is also seen with the intention to have a child vaccinated against HPV (peak at $80 \%$ in 2008 and now $41 \%$ in 2011).

In terms of barriers against the vaccine, parents still have safety and side-effect questions and they want more information. Parents view the vaccine like the oral contraceptive pill; it is best to invest in it only when you become at risk (i.e., you are sexually active). Parents who have high cancer 
worries and receive strong messages about HPV risks are more likely to advocate for the HPV vaccine. Parents look to their physicians to recommend the vaccine.

The strengths of this study are that it involves information gathered from a large number of parents from several countries. It shows trends in knowledge, attitudes, and behaviours over a time period just preceding the FDA approval of the vaccine; during the approval phase when there were extensive educational campaigns both by the pharmaceutical companies, professional societies, and media, after the FDA approval. The limitations of this study was an inability to validate parental responses, for example, determining how many parents had their child vaccinated with at least one dose of the vaccine.

It will be interesting to see if there are changes in parental attitudes as the types of information about HPV and the HPV vaccine continue to flood the literature. The information about the role of oncogenic HPV in more than cervical cancer is certainly evolving. We are just beginning to grasp the prevention implications of the HPV vaccine in the prevention of anal, oropharengeal, and a proportion of vulvovaginal, and penile cancers. The recent approval of the vaccine in young men may have an impact on decreasing condyloma transmission and having an impact on the rise of anal dysplasia/cancer in the male having sex with male population. As the cervical screening strategy moves toward primary HPV testing, this will also enhance education of the population. Although cost did not emerge as a significant barrier, as the vaccine prices continue to fall, it will be fascinating to see the impact on parental attitudes and behaviour. As public health looks at successful populationbased prevention strategies, it will be interesting to look at parental attitudes toward passive consent versus active consent in school-based vaccination programs. Time will provide information on how durable the vaccine is and longterm sequelae; whether this will influence parental attitudes remains to be seen.

In terms of future implications for policy, when the goal is to preserve the health of the population, certainly the passive consent approach, whether it is for vaccination or cervical screening, seems to be showing profound benefits. There is preliminary data that shows women who are vaccinated have less need for cervical precancer procedures like biopsies and treatment, however, how this will impact guidelines and availability of such services in the future remains to be seen.

\section{Conclusion}

Initial awareness of the virus and the ability of the virus to cause cancer have increased in the time period under study. However, awareness of the vaccine, intent to vaccinate, rates of vaccination rose during the initial introduction of the HPV vaccine but have fallen in subsequent years. Surveys have confirmed that parents want more knowledge and reassurance from their physicians that the HPV vaccine is safe for their children to receive. Policy programs, aimed at increasing HPV vaccination rates as part of an overall HPV strategy to reduce the incidents of cancers and infections caused by the virus, will need to heed the parents' concerns and information needs to be effective.

\section{References}

[1] J. M. M. Walboomers, M. V. Jacobs, M. M. Manos et al., "Human papillomavirus is a necessary cause of invasive cervical cancer worldwide," Journal of Pathology, vol. 189, no. 1, pp. 12-19, 1999.

[2] HPV Info, "What is HPV?" The Society of Obstetricians and Gynaecologists of Canada, Ottawa, Canada, 2009, http://hpvinfo.ca/hpvinfo/professionals/overview-2.aspx.

[3] HPV Info, "Incidence and prevalence in Canada," The Society of Obstetricians and Gynaecologists of Canada, Ottawa, Canada, 2009, http://hpvinfo.ca/hpvinfo/professionals/overview-3.aspx.

[4] K. A. Ault, A. R. Giuliano, R. P. Edwards et al., "A phase I study to evaluate a human papillomavirus (HPV) type 18 L1 VLP vaccine," Vaccine, vol. 22, no. 23-24, pp. 3004-3007, 2004.

[5] U.S. Food and Drug Administration, "Approved Products: Gardasil," U.S. Food and Drug Administration, Silver Spring, Md, USA, http://www.fda.gov/BiologicsBloodVaccines/Vaccines/ApprovedProducts/UCM094042.

[6] U.S. Food and Drug Administration, "Approved Products: Cervarix," U.S. Food and Drug Administration, Silver Spring, Md, USA, http://www.fda.gov/BiologicsBloodVaccines/Vaccines/ApprovedProducts/ucm186957.htm.

[7] An Advisory Committee Statement (ACS), National Advisory Committee on Immunization (NACI), "Statement on human papillomavirus vaccine," Public Health Agency of Canada, vol. 33, ACS-2, February 2007.

[8] Public Health Division, Ontario Ministry of Health and LongTerm Care, "Initial report on public health," Tech. Rep., Public Health Division, Ontario Ministry of Health and Long-Term Care, Toronto, Canada, August 2009.

[9] C. O. Mackie, J. A. Buxton, S. Tadwalkar, and D. M. Patrick, "Hepatitis B immunization strategies: timing is everything," Canadian Medical Association Journal, vol. 180, no. 2, pp. 196202, 2009.

[10] L. E. Markowitz, E. F. Dunne, M. Saraiya, H. W. Lawson, H. Chesson, and E. R. Unger, "Quadrivalent human papillomavirus vaccine: recommendations of the Advisory Committee on Immunization Practices (ACIP)," MMWRRecommendations and Reports, vol. 56, no. 2, pp. 1-24, 2007.

[11] J. D. Allen, M. K. D. Othus, R. C. Shelton et al., "Parental decision making about the HPV vaccine," Cancer Epidemiology Biomarkers and Prevention, vol. 19, no. 9, pp. 2187-2198, 2010.

[12] N. M. Askelson, S. Campo, J. B. Lowe, S. Smith, L. K. Dennis, and J. Andsager, "Using the theory of planned behavior to predict mothers' intentions to vaccinate their daughters against HPV," Journal of School Nursing, vol. 26, no. 3, pp. 194202, 2010.

[13] J. L. Barnack, D. M. Reddy, and C. Swain, "Predictors of parents' willingness to vaccinate for human papillomavirus and physicians' intentions to recommend the vaccine," Women's Health Issues, vol. 20, no. 1, pp. 28-34, 2010.

[14] D. H. Bernat, S. B. Harpin, M. E. Eisenberg, L. H. Bearinger, and M. D. Resnick, "Parental support for the human papillomavirus vaccine," Journal of Adolescent Health, vol. 45, no. 5, pp. 525-527, 2009. 
[15] L. Brabin, S. A. Roberts, F. Farzaneh, and H. C. Kitchener, "Future acceptance of adolescent human papillomavirus vaccination: a survey of parental attitudes," Vaccine, vol. 24, no. 16, pp. 3087-3094, 2006.

[16] C. R. Breitkopf, H. C. Pearson, T. A. Dinh et al., "Human papillomavirus vaccine decision-making in Da Nang, Vietnam: perceived spousal and adolescent-parent concordance," Vaccine, vol. 27, no. 17, pp. 2367-2371, 2009.

[17] N. T. Brewer, S. L. Gottlieb, P. L. Reiter et al., "Longitudinal predictors of human papillomavirus vaccine initiation among adolescent girls in a high-risk geographic area," Sexually Transmitted Diseases, vol. 38, no. 3, pp. 197-204, 2011.

[18] D. S. Brown, F. R. Johnson, C. Poulos, and M. L. Messonnier, "Mothers' preferences and willingness to pay for vaccinating daughters against human papillomavirus," Vaccine, vol. 28, no. 7, pp. 1702-1708, 2010.

[19] J. R. Cates, A. Shafer, F. D. Carpentier et al., "How parents hear about human papillomavirus vaccine: implications for uptake," Journal of Adolescent Health, vol. 47, no. 3, pp. 305308, 2010.

[20] S. S. Chan, T. H. Cheung, W. K. Lo, and T. K. H. Chung, "Women's attitudes on human papillomavirus vaccination to their daughters," Journal of Adolescent Health, vol. 41, no. 2, pp. 204-207, 2007.

[21] S. N. Chow, R. Soon, J. S. Park et al., "Knowledge, attitudes, and communication around human papillomavirus (HPV) vaccination amongst urban Asian mothers and physicians," Vaccine, vol. 28, no. 22, pp. 3809-3817, 2010.

[22] N. A. Constantine and P. Jerman, "Acceptance of human papillomavirus vaccination among Californian parents of daughters: a representative statewide analysis," Journal of Adolescent Health, vol. 40, no. 2, pp. 108-115, 2007.

[23] L. A. Dahlstrom, T. N. Tran, C. Lundholm, C. Young, K. Sundstrom, and P. Sparen, "Attitudes to HPV vaccination among parents of children aged 12-15 years-a populationbased survey in Sweden," International Journal of Cancer, vol. 126, no. 2, pp. 500-507, 2010.

[24] K. Davis, E. D. Dickman, D. Ferris, and J. K. Dias, "Human papillomavirus vaccine acceptability among parents of 10to 15-year-old adolescents," Journal of Lower Genital Tract Disease, vol. 8, no. 3, pp. 188-194, 2004.

[25] R. de Visser and E. McDonnell, “Correlates of parents' reports of acceptability of human papilloma virus vaccination for their school-aged children," Sexual Health, vol. 5, no. 4, pp. 331$338,2008$.

[26] A. F. Dempsey, L. M. Abraham, V. Dalton, and M. Ruffin, "Understanding the reasons why mothers do or do not have their adolescent daughters vaccinated against human papillomavirus," Annals of Epidemiology, vol. 19, no. 8, pp. 531-538, 2009.

[27] A. F. Dempsey, G. D. Zimet, R. L. Davis, and L. Koutsky, "Factors that are associated with parental acceptance of human papillomavirus vaccines: a randomized intervention study of written information about HPV," Pediatrics, vol. 117, no. 5, pp. 1486-1493, 2006.

[28] T. A. Dinh, S. L. Rosenthal, E. D. Doan et al., "Attitudes of mothers in Da Nang, Vietnam toward a human papillomavirus vaccine," Journal of Adolescent Health, vol. 40, no. 6, pp. 559-563, 2007.

[29] P. Dursun, B. Altuntas, E. Kuscu, and A. Ayhan, "Women's knowledge about human papillomavirus and their acceptance of HPV vaccine," Australian and New Zealand Journal of Obstetrics and Gynaecology, vol. 49, no. 2, pp. 202-206, 2009.
[30] C. Y. Fang, E. J. Coups, and C. J. Heckman, "Behavioral correlates of HPV vaccine acceptability in the 2007 Health Information National Trends Survey (HINTS)," Cancer Epidemiology Biomarkers and Prevention, vol. 19, no. 2, pp. 319326,2010 .

[31] D. Ferris, L. Horn, and J. L. Waller, "Parental acceptance of a mandatory human papillomavirus (HPV) vaccination program," Journal of the American Board of Family Medicine, vol. 23, no. 2, pp. 220-229, 2010.

[32] M. A. Gerend, E. Weibley, and H. Bland, "Parental response to human papillomavirus vaccine availability: uptake and intentions," Journal of Adolescent Health, vol. 45, no. 5, pp. 528-531, 2009.

[33] L. Gillespie, C. W. Hicks, M. Santana et al., "The acceptability of human papillomavirus vaccine among parents and guardians of newborn to 10-year-old children," Journal of Pediatric and Adolescent Gynecology, vol. 24, no. 2, pp. 66-70, 2011.

[34] S. L. Gottlieb, N. T. Brewer, M. R. Sternberg et al., "Human papillomavirus vaccine initiation in an area with elevated rates of cervical cancer," Journal of Adolescent Health, vol. 45, no. 5, pp. 430-437, 2009.

[35] S. L. Guerry, C. J. De Rosa, L. E. Markowitz et al., "Human papillomavirus vaccine initiation among adolescent girls in high-risk communities," Vaccine, vol. 29, no. 12, pp. 22352241, 2011.

[36] K. Hausdorf, B. Newman, D. Whiteman, J. Aitken, and I. Frazer, "HPV vaccination: what do Queensland parents think?" Australian and New Zealand Journal of Public Health, vol. 31, no. 3, pp. 288-289, 2007.

[37] L. Horn, C. Howard, J. Waller, and D. G. Ferris, "Opinions of parents about school-entry mandates for the human papillomavirus vaccine," Journal of Lower Genital Tract Disease, vol. 14, no. 1, pp. 43-48, 2010.

[38] J. Hughes, J. R. Cates, N. Liddon, J. S. Smith, S. L. Gottlieb, and N. T. Brewer, "Disparities in how parents are learning about the human papillomavirus vaccine," Cancer Epidemiology Biomarkers and Prevention, vol. 18, no. 2, pp. 363-372, 2009.

[39] E. Ilter, A. Celik, B. Haliloglu et al., "Women's knowledge of Pap smear test and human papillomavirus: acceptance of HPV vaccination to themselves and their daughters in an islamic society," International Journal of Gynecological Cancer, vol. 20, no. 6, pp. 1058-1062, 2010.

[40] J. A. Kahn, L. Ding, B. Huang, G. D. Zimet, S. L. Rosenthal, and A. L. Frazier, "Mothers' intention for their daughters and themselves to receive the human papillomavirus vaccine: a national study of nurses," Pediatrics, vol. 123, no. 6, pp. 14391445, 2009.

[41] H. S. Kang and L. Moneyham, "Attitudes, intentions, and perceived barriers to human papillomavirus vaccination among Korean high school girls and their mothers," Cancer Nursing, vol. 34, no. 3, pp. 202-208, 2011.

[42] C. H. Lenselink, M. M. Gerrits, W. J. Melchers, L. F. Massuger, D. van Hamont, and R. L. Bekkers, "Parental acceptance of human papillomavirus vaccines," European Journal of Obstetrics and Gynecology and Reproductive Biology, vol. 137, no. 1, pp. 103-107, 2008.

[43] L. A. Marlow, A. S. Forster, J. Wardle, and J. Waller, "Mothers' and adolescents' beliefs about risk compensation following HPV vaccination," Journal of Adolescent Health, vol. 44, no. 5, pp. 446-451, 2009.

[44] L. A. Marlow, J. Waller, and J. Wardle, "Sociodemographic predictors of HPV testing and vaccination acceptability: results from a population-representative sample of British 
women," Journal of Medical Screening, vol. 15, no. 2, pp. 9196, 2008.

[45] L. A. Marlow, J. Waller, and J. Wardle, "Trust and experience as predictors of HPV vaccine acceptance," Human Vaccines, vol. 3, no. 5, pp. 171-175, 2007.

[46] H. Marshall, P. Ryan, D. Roberton, and P. Baghurst, "A crosssectional survey to assess community attitudes to introduction of human papillomavirus vaccine," Australian and New Zealand Journal of Public Health, vol. 31, no. 3, pp. 235-242, 2007.

[47] G. L. Mortensen, "Parental attitudes towards vaccinating sons with human papillomavirus vaccine," Danish Medical Bulletin, vol. 57, no. 12, article A4230, 2010.

[48] G. Ogilvie, M. Anderson, F. Marra et al., "A population-based evaluation of a publicly funded, school-based HPV vaccine program in British Columbia, Canada: parental factors associated with HPV vaccine receipt," PLoS Medicine, vol. 7, no. 5, article e1000270, 2010.

[49] G. S. Ogilvie, V. P. Remple, F. Marra et al., "Intention of parents to have male children vaccinated with the human papillomavirus vaccine," Sexually Transmitted Infections, vol. 84, no. 4, pp. 318-323, 2008.

[50] C. Pelucchi, S. Esposito, C. Galeone et al., "Knowledge of human papillomavirus infection and its prevention among adolescents and parents in the greater Milan area, Northern Italy," BMC Public Health, vol. 10, article 378, 2010.

[51] R. Podolsky, M. Cremer, J. Atrio, T. Hochman, and A. A. Arslan, "HPV vaccine acceptability by Latino parents: a comparison of U.S. and Salvadoran populations," Journal of Pediatric and Adolescent Gynecology, vol. 22, no. 4, pp. 205215, 2009.

[52] C. M. Rand, S. J. Schaffer, S. G. Humiston et al., "Patientprovider communication and human papillomavirus vaccine acceptance," Clinical Pediatrics, vol. 50, no. 2, pp. 106-113, 2011.

[53] P. L. Reiter, N. T. Brewer, S. L. Gottlieb, A. L. McRee, and J. S. Smith, "Parents' health beliefs and HPV vaccination of their adolescent daughters," Social Science and Medicine, vol. 69, no. 3, pp. 475-480, 2009.

[54] P. L. Reiter, J. R. Cates, A. L. McRee et al., "Statewide HPV vaccine initiation among adolescent females in North Carolina," Sexually Transmitted Diseases, vol. 37, no. 9, pp. 549-556, 2010.

[55] P. L. Reiter, A. L. McRee, S. L. Gottlieb, and N. T. Brewer, "Correlates of receiving recommended adolescent vaccines among adolescent females in North Carolina," Human Vaccines, vol. 7, no. 1, pp. 67-73, 2011.

[56] P. L. Reiter, A. L. McRee, S. L. Gottlieb, and N. T. Brewer, "HPV vaccine for adolescent males: acceptability to parents postvaccine licensure," Vaccine, vol. 28, no. 38, pp. 6292-6297, 2010.

[57] S. B. Rose, B. A. Lawton, T. Lanumata, M. Hibma, and M. G. Baker, "HPV/cervical cancer vaccination: parental preferences on age, place and information needs," Journal of Primary Health Care, vol. 2, no. 3, pp. 190-198, 2010.

[58] S. L. Rosenthal, R. Rupp, G. D. Zimet et al., "Uptake of HPV vaccine: demographics, sexual history and values, parenting style, and vaccine attitudes," Journal of Adolescent Health, vol. 43, no. 3, pp. 239-245, 2008.

[59] M. Toffolon-Weiss, K. Hagan, J. Leston, L. Peterson, E. Provost, and T. Hennessy, "Alaska Native parental attitudes on cervical cancer, HPV and the HPV vaccine," International Journal of Circumpolar Health, vol. 67, no. 4, pp. 363-373, 2008.
[60] A. E. Tozzi, L. Rava, D. Stat, E. Pandolfi, M. G. Marino, and A. G. Ugazio, "Attitudes towards HPV immunization of Italian mothers of adolescent girls and potential role of health professionals in the immunization program," Vaccine, vol. 27, no. 19, pp. 2625-2629, 2009.

[61] S. C. Woodhall, M. Lehtinen, T. Verho, H. Huhtala, M. Hokkanen, and E. Kosunen, "Anticipated acceptance of HPV vaccination at the baseline of implementation: a survey of parental and adolescent knowledge and attitudes in Finland," Journal of Adolescent Health, vol. 40, no. 5, pp. 466-469, 2007.

[62] N. Yeganeh, D. Curtis, and A. Kuo, "Factors influencing HPV vaccination status in a Latino population; and parental attitudes towards vaccine mandates," Vaccine, vol. 28, no. 25, pp. 4186-4191, 2010.

[63] K. L. Ziarnowski, N. T. Brewer, and B. Weber, "Present choices, future outcomes: anticipated regret and HPV vaccination," Preventive Medicine, vol. 48, no. 5, pp. 411-414, 2009. 


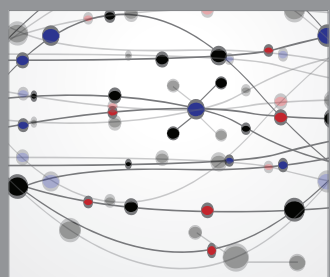

The Scientific World Journal
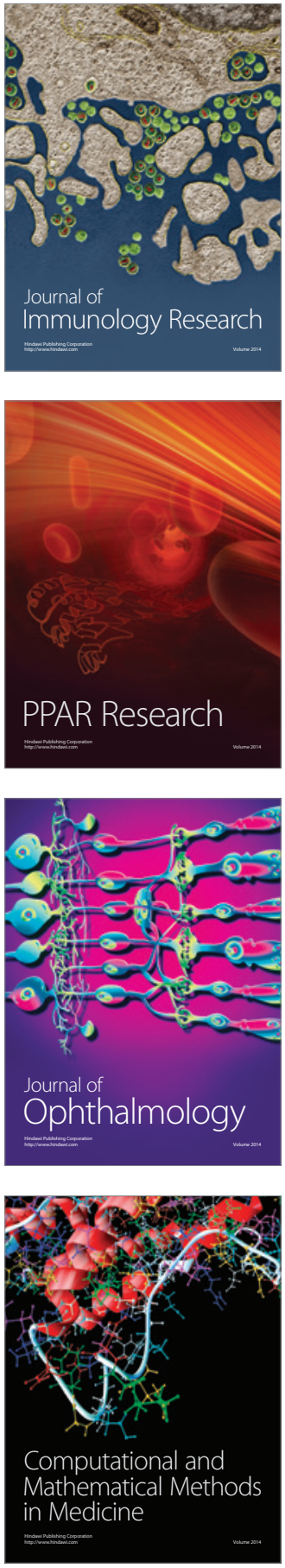

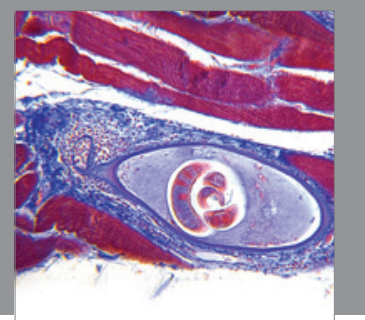

Gastroenterology

Research and Practice
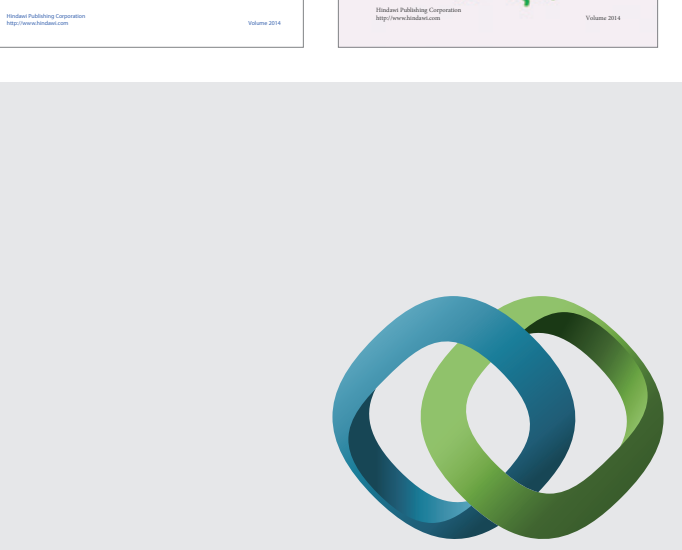

\section{Hindawi}

Submit your manuscripts at

http://www.hindawi.com
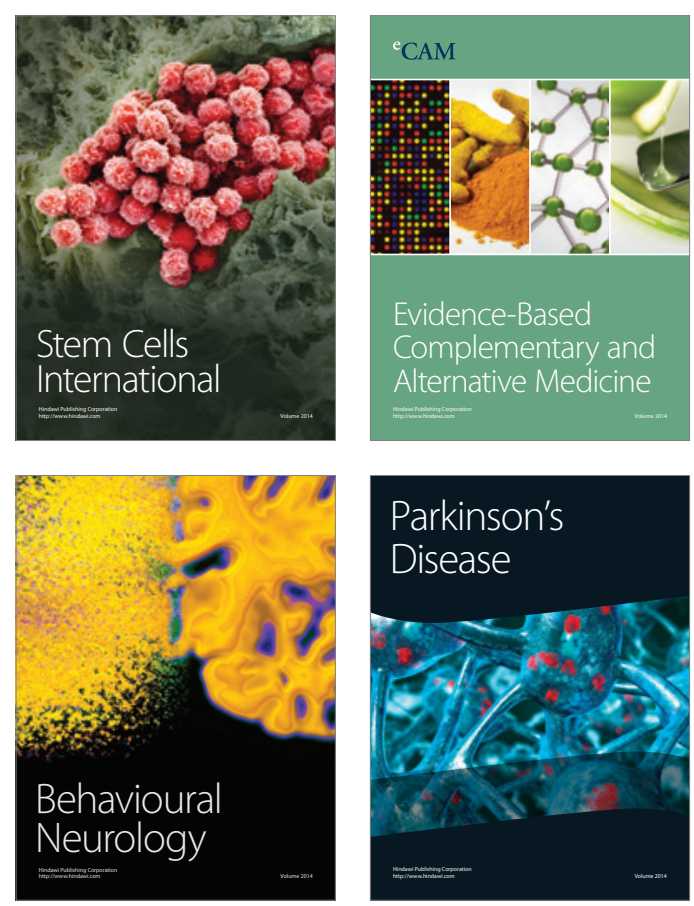

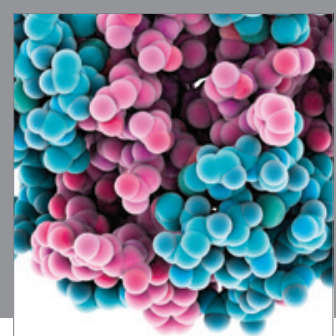

Journal of
Diabetes Research

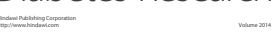

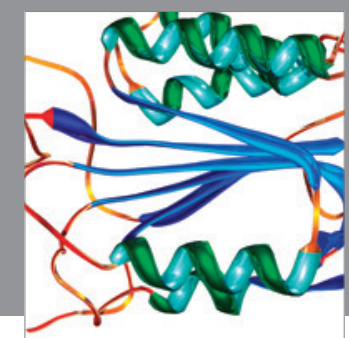

Disease Markers
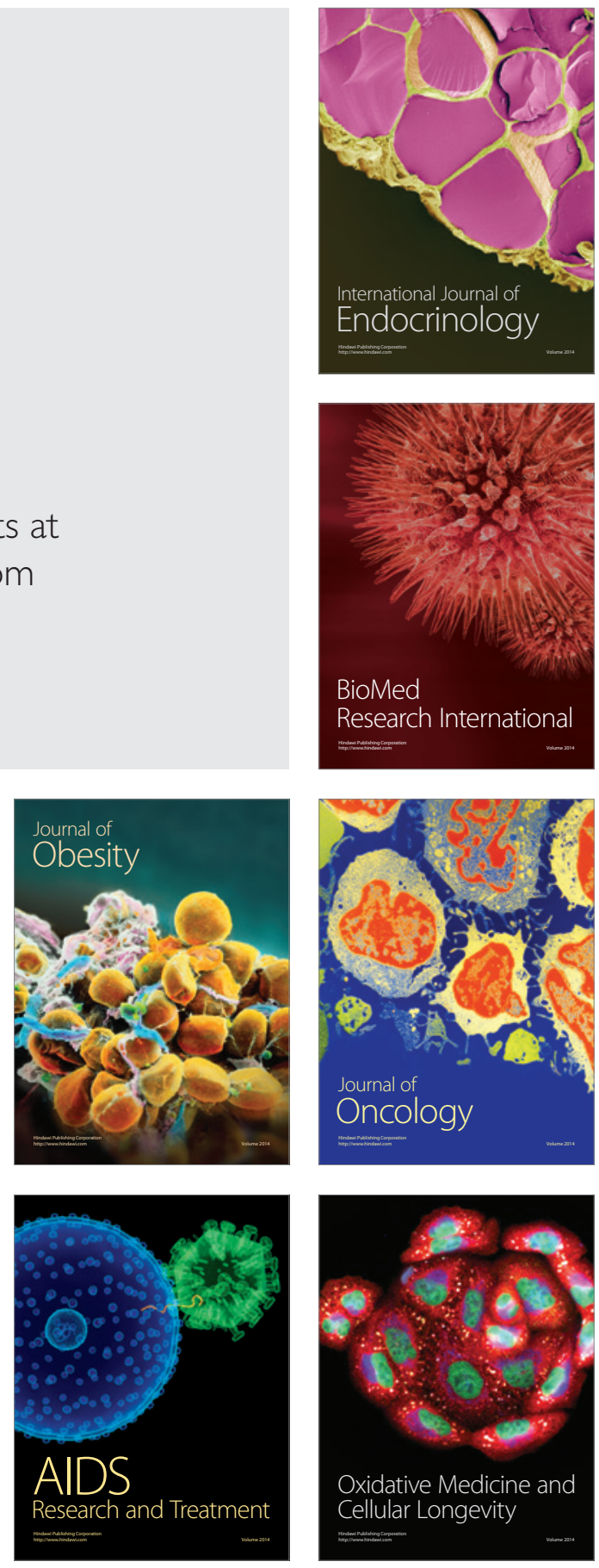